\title{
Metabolic adaptation and neuroprotection differ in the retina and choroid in a piglet model of acute postnatal hypoxia
}

\author{
Alessandro Arduini',2, Javier Escobar ${ }^{3}$, Maximo Vento ${ }^{3,4}$, Raquel Escrig ${ }^{4}$, Guillermo Quintás ${ }^{5}$, Juan Sastre', Ola Didrik Saugstad ${ }^{6}$ \\ and Rønnaug Solberg ${ }^{6}$
}

\begin{abstract}
BACKGROUND: Hypoxic-ischemic insults to the neonatal brain may cause neurodevelopmental disorders. Vulnerability of different areas of the neural tissue to hypoxic-ischemic stress might be explained by either heterogeneous sensitivity to oxygen or neuroprotective capability. Our understanding of regional heterogeneity is still incomplete in terms of metabolic reconfiguration and/or activation of neuroprotective mechanisms.
\end{abstract}

METHODS: We studied, by western blotting, reversetranscriptase $P C R$, and tandem mass spectrometry, the response of retina and choroid at protein, gene, and metabolic levels during hypoxia in a piglet model of acute postnatal hypoxia.

RESULTS: We evidenced a metabolic shift towards glycolysis in choroid after hypoxia while retina experienced a dramatic energy stress with decreased mitochondrial metabolites. Hypoxia-inducible transcription factor-1a (HIF-1a) was not stabilized in retina during hypoxia, supported by a deficient signaling from $v$-akt murine thymoma viral oncogene (AKT) and ERK1/2, and unchanged glutathione redox status. In retina, but not in choroid, phosphorylation of p65 (NF-kB) and increased transcription of target genes may have a major role during hypoxic stress.

CONCLUSION: We showed that the retina engages a distinct pattern of signaling and transcriptional events than observed in the choroid. Retina and choroid may reflect regional sensitivity to hypoxia. While prolonged and intense hypoxia may jeopardize retinal cell survival, choroid sets up a different pattern of response, which promotes adaptation to these adverse conditions.

$F_{\text {it }}^{\text {etong }}$ tal-to-neonatal transition is characterized by a rapid increase in oxygen delivered to tissue. Hence, while in utero arterial oxygen saturation as measured by pulse oximetry is around $35-55 \%$, only few minutes after birth pulse oximetry will abruptly rise to $>90 \%$ increasing tissue oxygenation and subsequent oxidative stress that contributes to the physiology of postnatal adaptation (1). However, pathophysiological conditions, such as prematurity or birth asphyxia, with or without need of resuscitation, exacerbate oxidative stress, and enhance inflammation (1). Under these circumstances especially sensitive tissues, such as the retina and choroid are vulnerable towards damage. It is therefore important to define a safety range of postnatal oxygenation that would avoid damage caused by both hyper-and/or-hypoxia in the immediate postnatal period (2), and thereafter (1).

Interestingly, blood supply and oxygen are delivered to the retina by two different vascular systems-the uveal and the retinal. The inner choroidal vasculature is mainly controlled by sympathetic innervations and its flow is in excess to its metabolic needs. Contrarily, retinal circulation is regulated by local factors, such as mechanical pressure, oxygen tension, and $\mathrm{pH}$ (3). Of note, retinal metabolism is one of the highest in the human organism (4), and retina is considered one of the most $\mathrm{O}_{2}$-sensitive tissues (5). Requirements of oxygen and nutrients might pose a serious challenge during prolonged hypoxic and/or ischemic periods. Under normoxic conditions, the choroidal vasculature has higher oxygen content than the retinal vasculature (6), and severe hypoxia largely reduces retinal blood flow (7). Herewith, the intrinsic differences in blood supply and metabolism between retina and choroid may induce a different type of response to hypoxic-ischemic stress.

Mechanisms of neuroprotection are central to preserve physiology during hypoxia or after hypoxic/ischemic brain injury in retinal cells (8). Activation of hypoxia-inducible transcription factors (HIF) is one of the main mechanisms for cells to adapt to low $\mathrm{O}_{2}$ concentration (8). Metabolic reconfiguration (inhibition of Krebs's cycle and activation of glycolysis) and enhancement of $\mathrm{O}_{2}$ delivery by angiogenesis and vasodilatation are essential components of an adequate physiologic response $(9,10)$. Under physiologic oxygen availability, HIF- $\alpha$ subunits $(1 \alpha, 2 \alpha$, and $3 \alpha)$ are regulated at posttranslational level by prolyl-hydroxylases and also the factor inhibiting HIF-1 that, respectively, fosters $\alpha$ subunits degradation and inactivation. They are both using oxygen and $\alpha$-ketoglutarate

\footnotetext{
'Department of Physiology, Faculty of Pharmacy, University of Valencia, Valencia, Spain; ${ }^{2}$ Department of Genetics and Complex Diseases, Harvard School of Public Health, Boston, Massachusetts; ${ }^{3}$ Neonatal Research Unit, Health Research Institute La Fe, Valencia, Spain; ${ }^{4}$ Division of Neonatology, University and Polytechnic Hospital La Fe, Valencia, Spain; 5 Leitat Technological Center, Bio In Vitro Division, Barcelona, Spain; ${ }^{6}$ Department of Pediatric Research, Institute for Surgical Research, Oslo University Hospital-Rikshospitalet, Oslo, Norway. Correspondence: Rønnaug Solberg (ronnaug.solberg@medisin.uio.no) 
Table 1. Characterization of the study cohort

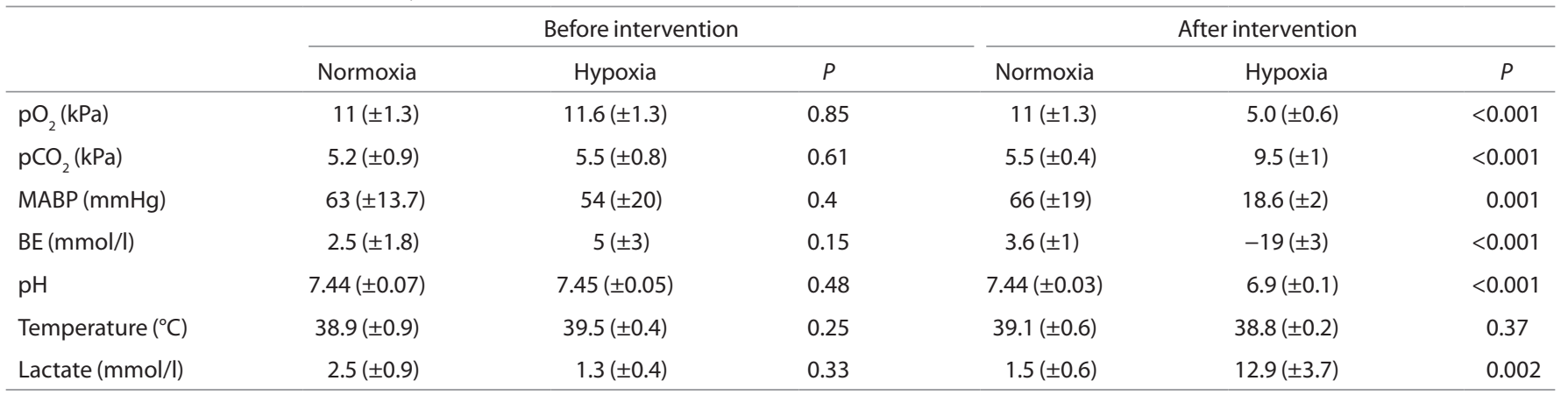

Characterization of the study cohort before and after asphyxia, or equivalent sham procedure. Hypoxia induced significant changes in pH, lactate, base excess, and mean arterial blood pressure in a similar extend between the groups of animals that underwent hypoxia. Tukey's test was used for data analysis.

as substrates $(10,11)$. On the contrary, during hypoxia, stabilization and HIF- $1 \alpha$ transactivation activity is promoted by an array of signaling events like mitogen-activated protein kinase 3/1, ERK1/2, mechanistic target of rapamycin (MTOR), and AKT $(12,13)$. HIF-1 $\alpha$, which plays a major role during acute episodes of hypoxia (14) and its canonical target vascular endothelial growth factor alpha (VEGFA), involved in angiogenesis and vascular remodeling, exert neuroprotection during cerebral hypoxia-ischemia $(8,15)$. Both HIF- $1 \alpha$ and VEGF- $\alpha$ levels are altered in a number of retinal disorders, such as hypoxia/ischemia and retinopathy of prematurity and experimental evidence supports its role in promoting pathological angiogenesis $(16,17)$. Intriguingly, at present it is unclear if a pathological state is favored by HIF-1 $\alpha$ itself or by one of its target genes, like VEGFA. Other transcription factors, like $\mathrm{NF}-\kappa \mathrm{B}$ and signal transducers and activators of transcription 3, can also drive VEGFA transcription during hypoxic stress $(18,19)$.

The different metabolic and blood flow regulation characteristics of retina and choroid could therefore be a paradigm of cerebral blood flow in the extremely preterm infant. Hence, it has been previously shown that preterm infants have poorly developed autoregulation mechanisms in response to changes in arterial blood pressure and/or arterial oxygen content, as is the case in choroid (20). Taking this hypothesis into consideration, our aim was to characterize and compare the biochemical and molecular adaptive responses to acute postnatal hypoxia in both tissues. Our results show that retina and choroid have a different biochemical and molecular pattern in response to hypoxic stress. Therefore, choroidal metabolic adaptive mechanisms could reflect the pathophysiologic consequences in the preterm brain under different oxygenation conditions and serve as an experimental model for translational purposes (20).

\section{RESULTS}

\section{Cohort Characterization During the Experiment}

The summary of the cohort characteristics before and after hypoxia is given in Table 1. Hypoxia lasted on average of $68 \pm 15 \mathrm{~min}(n=5)$, and induced significant changes in $\mathrm{pO}_{2}$, $\mathrm{pCO}_{2}$, mean arterial blood pressure, $\mathrm{pH}$, base excess, and lactate (Tukey's adjusted, $P \leq 0.002$ ).

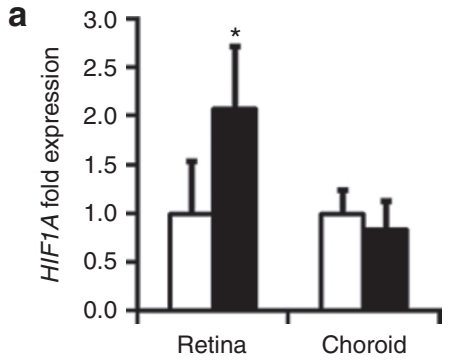

b

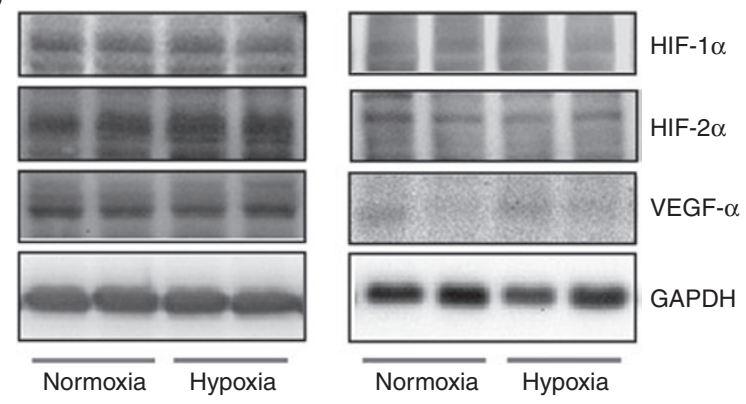

Figure 1. Regulation of HIF after hypoxia in retina and choroid. Hypoxiainducible transcription factor (HIF)- $1 \alpha$ is regulated at transcriptional level but not its protein abundance after hypoxic challenge. (a) HIF-1A mRNA was increased in retina in hypoxia vs. normoxia $(P<0.05)$, and no change was observed in choroid. Glyceraldehyde 3-phosphate dehydrogenase (GAPDH) mRNA level was used in reverse-transcriptase PCR experiments as an internal control. (b) Protein levels of HIF- $1 \alpha$, HIF- $2 \alpha$, and VEGF- $\alpha$ in retina (left) and choroid (right) from normoxia and hypoxia groups of piglets ( $P>0.05$ for all comparisons, hypoxia vs. normoxia). GAPDH was used as a loading control. Student's $t$-test was used for statistical comparisons. ${ }^{*} P<0.05$. White bars represent normoxia; black bars represent hypoxia. VEGF, vascular endothelial growth factor.

\section{HIF-1 $\alpha$ Activation in Retina and Choroid Following Hypoxia}

Regulation and activity of HIF- $1 \alpha$ in retina and choroid were studied. Hypoxia promoted an increase in HIF-1a mRNA in retina $(P<0.05)$, but not in choroid (Figure 1$)$. Nevertheless, we did not detect HIF- $1 \alpha$ or HIF- $2 \alpha$ stabilization or regulation of VEGF- $\alpha$ at protein level by western blotting neither in retina or choroid (Figure 1). Since the half-life of HIF- $1 \alpha$ protein can be as short as $\sim 4-6$ min (21) we reasoned that degradation of HIF- $1 \alpha$ might occur during the procedure of tissue isolation. Thus, we studied the phosphorylation level of MTOR, AKT, and ERK1/2, critical 
a

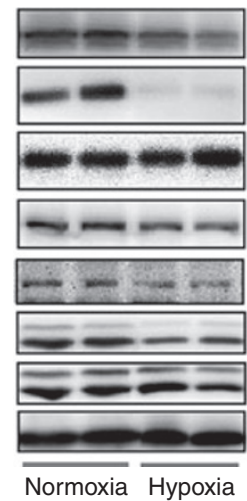

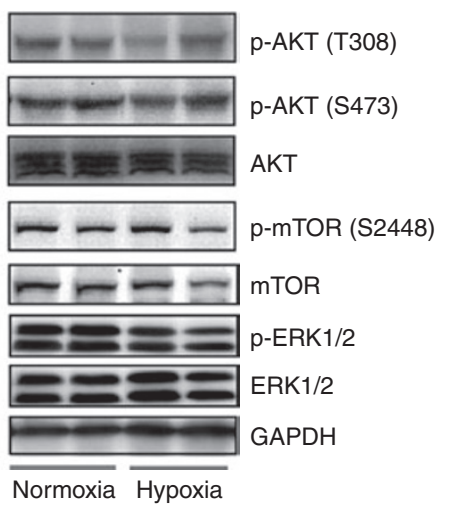

d

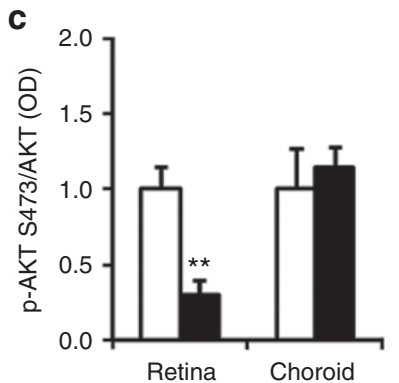

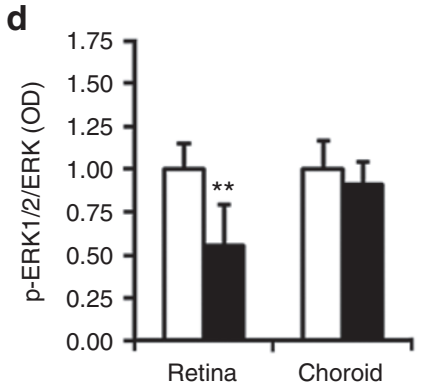

e

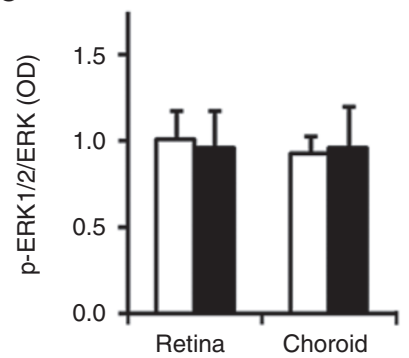

Figure 2. Signaling pathway that regulates hypoxia-inducible transcription factors (HIF) protein stabilization and/or transcriptional activation. Retina and choroid show a different pattern of activation of stress kinases that promote HIF stabilization and activation. (a) In retina (left), ERK1/2, and v-akt murine thymoma viral oncogene (AKT) are significantly dephosphorylated in response to hypoxia. In choroid (right), no change in AKT and ERK1/2 phosphorylation was observed. (b-e) Densitometric quantification of protein phosphorylation of AKT(T308), AKT(S473), ERK1/2, MTOR(S2448) in retina and choroid. ${ }^{*} P<0.05,{ }^{* *} P<0.01$ White bars represent normoxia; black bars represent hypoxia.

kinases in the stabilization of HIF-1 $\alpha$. We did not observe any significant change in MTOR status, but we observed a marked decrease in both AKT and ERK1/2 in retina $(P<$ 0.01 and $P<0.05$, respectively), and no change in choroid (Figure 2).

We measured the expression level of a subset of HIF-1 $\alpha$ signature target genes (Figure 3), relevant to our study, as readout of its activity (12). In retina, we observed an increase in VEGFA $(P<0.05)$, adrenomedullin $(A D M, P<$ 0.01 ), and phosphoglycerate kinase $1(P G K 1, P<0.05)$, but no change in several other genes. In choroid, VEGFA was increased $(P<0.01)$, but aldolase $\mathrm{C}(A L D O C)$ was markedly repressed $(P<0.01)$.

\section{Regulation of Stress Response and Survival Pathways}

We found that the subunit p65 of NF- $\mathrm{\kappa B}$ was more phosphorylated in retina in response to hypoxia $(P<0.05)$, but no change was observed in choroid (Figure 4). Conversely, signal transducers and activators of transcription 3 (STAT3) were less phosphorylated in retina in response to hypoxia, $P<0.05$ (Figure 4). Mitogen-activated protein kinase 11/12/13/14 (p38) kinase promotes brain-derived neurotrophic factor (BDNF) synthesis and secretion in microglial cells (22). In this study, p38 phosphorylation level was unchanged in retina and choroid following postnatal hypoxia. Accordingly, our results showed no change in BDNF mRNA level (Figure 5).
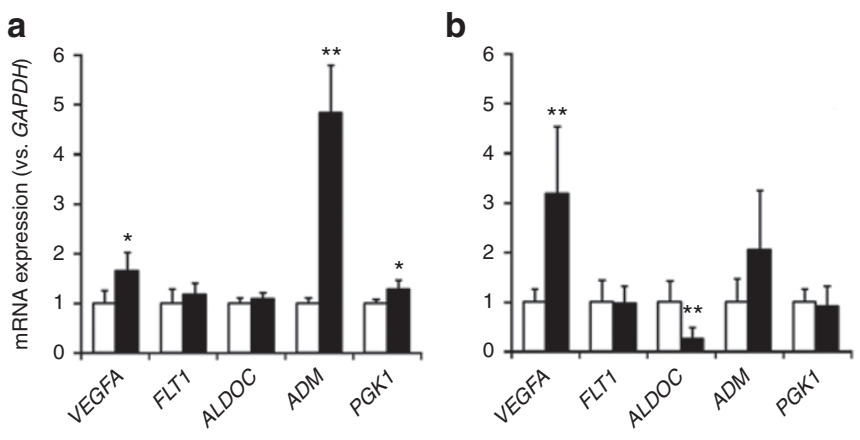

Figure 3. Gene expression in retina and choroid after hypoxia. Gene expression of HIF target genes and genes required for angiogenesis and vascular permeability reveals a specific response to hypoxia in (a) retina and (b) choroid. Gene expression was measured by reverse-transcriptase PCR. GAPDH mRNA was used as internal control. Student's $t$-test was used for statistical comparisons. ${ }^{*} P<0.05,{ }^{* *} P<0.01$. White bars represent normoxia; black bars represent hypoxia. ADM, adrenomedullin; $A L D O C$, aldolase $C$; Flt-1, fms-related tyrosine kinase $1 ; P G K 1$, phosphoglycerate kinase $1 ;$ VEGFA, vascular endothelial growth factor $\alpha$.

Metabolic Response to Postnatal Hypoxia in Retina and Choroid We used a target mass spectrometry approach to assess levels of several metabolites of the energy metabolism. Our analyses showed that tissue acidosis (lactate/pyruvate ratio) was present in both retina $(P<0.01)$ and choroid $(P<0.05$, Figure 6$)$. Nonetheless, glucose-6-P and pyruvate, and several metabolites of the tricarboxylic acid cycle (isocitrate, $\alpha$-ketoglutarate, 
a
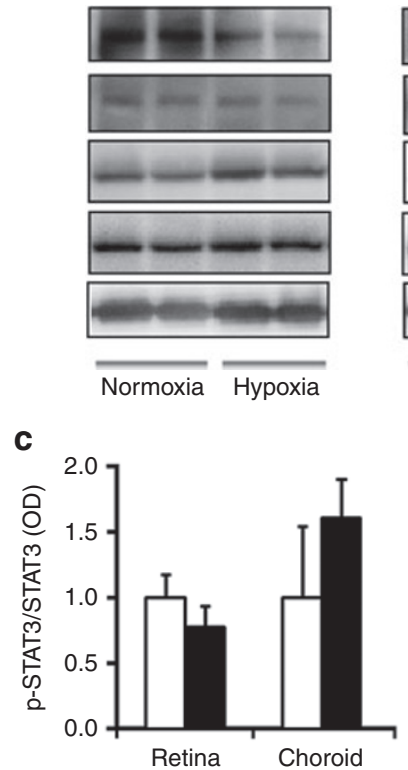

Figure 4. Signal transducers and activators of transcription 3 (STAT3) and NF-KB phosphorylation in response to hypoxia. Phosphoprotein and total protein of STAT3 and p65 (NF-KB) in (a) retina and (b) choroid after hypoxia. GAPDH was used as a loading control. (c) Densitometric quantification of STAT3 phosphorylation normalized to STAT3 total protein in retina and choroid in response to hypoxia. (d) Densitometric quantification of p65 (NF- $\mathrm{KB}$ ) phosphorylation normalized to $\mathrm{p} 65$ total protein in retina and choroid in response to hypoxia. ${ }^{*} P<0.05$. White bars represent normoxia; black bars represent hypoxia.

and malate) were markedly decreased in retina but not in choroid (Figure 6). Pentose phosphate pathway intermediates were unchanged in both tissues. A summary of all metabolites levels is presented in Supplementary Table S1 online.

Considering that nutrient stress, like glucose deprivation, can activate AMP-activated protein kinase, we measured total AMP-activated protein kinase (AMPK) level and its active form, p-AMPK T172, by immunoblotting. We did not observe AMPK activation in retina or choroid during hypoxia (Supplementary Figure S1 online).

\section{DISCUSSION}

In this study, we have discovered fundamental differences in choroideal and retinal tissues during baseline conditions and in response to hypoxia. There was a metabolic shift toward glycolysis in choroid after hypoxia while retina experienced a change in cellular bioenergetics with a reduction in mitochondrial metabolites.

Perinatal hypoxia ischemia is one of the leading causes of neurodevelopmental delay in infancy and childhood $(1,2)$. Regional vulnerability might be explained by a different ability for neuroprotection or sensitivity to oxygen metabolism of the affected areas. In addition, anatomical organization of retina and choroid may cause a more rapid drop in $\mathrm{pO} 2$ in the retina, which can itself predispose this tissue to a more severe damage. Herewith, we show that hypoxia triggers in retina and choroid a profound remodeling of cellular metabolism and a

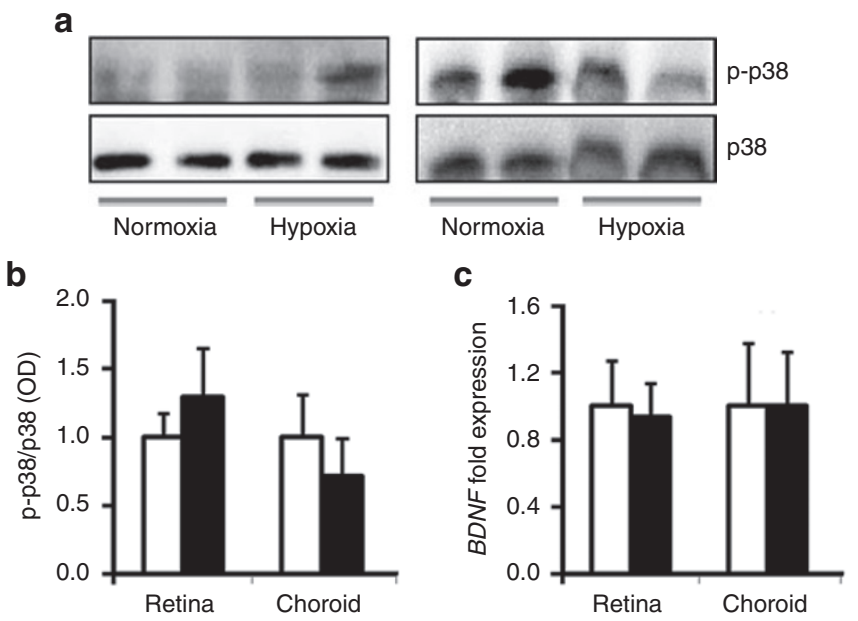

Figure 5. Neuroprotection in retina and choroid after hypoxia. (a) Activation status of p38 (p38 and total p38) in retina (left) and choroid (right) in normoxia and hypoxia. (b) Densitometric quantification of phosphorylated p38 and total p38 in western blot experiments. No statistical difference was found between hypoxia and normoxia $(P>0.5)$. (c) mRNA expression of BDNF in normoxia and hypoxia groups in retina and choroid. GAPDH was used as an internal control. No differences were found in retina or choroid. Student's $t$-test was used for statistical comparisons. White bars represent normoxia; black bars represent hypoxia.

stress response, yet uncoupled from the activation of neuroprotective mechanisms. Retina experienced a more dramatic metabolic collapse and stress response to hypoxia. It is at present unclear whether retina is incapable of a metabolic switch to increased glycolysis, or the degree of hypoxia is more severe in retina than in choroid. Despite this question is physiologically relevant, from a pathological perspective, we observed that the ability of the retinal tissue to adapt to hypoxic episodes is readily insufficient.

HIF-1 $\alpha$ plays a role in several retinal disorders $(16,17)$. Nevertheless, our results do not substantiate a role for HIF- $1 \alpha$ after hypoxia supported by several lines of evidence: (i) lack of HIF-1 $\alpha$ stabilization, (ii) signaling events that trigger HIF$1 \alpha$ activation were not activated in choroid and even repressed in retina (10-13). Gene expression analysis of HIF targets (ALDOC, PGK1, VEGFA, and ADM), did not match a clear HIF signature. Of note, $A L D O C$ was markedly decreased after hypoxia in choroid.

Several studies have shown that HIF- $1 \alpha$ protein can increase immediately after hypoxic exposure, and elevated levels persist up to $24 \mathrm{~h}$ after an insult (8). Nevertheless, exposure to both hypoxia and ischemia causes less HIF- $1 \alpha$ stabilization than severe hypoxia alone (23). Despite our model being one of hypoxia, a drastic drop in mean arterial blood pressure to 20 mmHg supports the idea of an ensuing metabolic ischemia. In similar experimental conditions, piglets challenged with hypoxia alone experience a marked reduction in cardiac output, which parallels a drop in $\mathrm{pH}$, oxygenation and mean arterial blood pressure (24). Thus, it is reasonable to assume that our model has features of both hypoxia and ischemia, a physiological condition that can contribute to a blunted stabilization of HIF- $1 \alpha(23)$. 
a

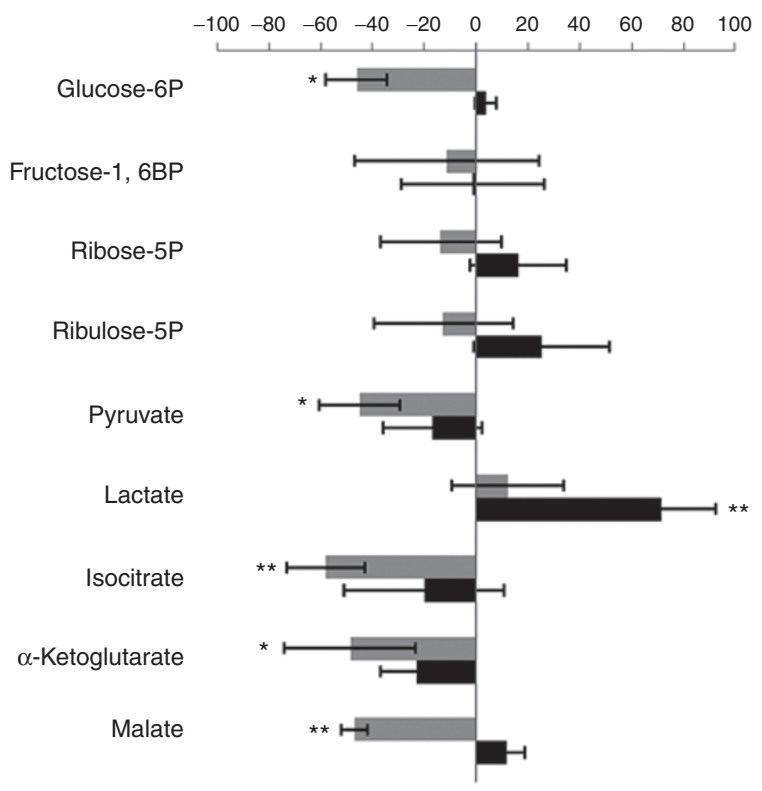

b

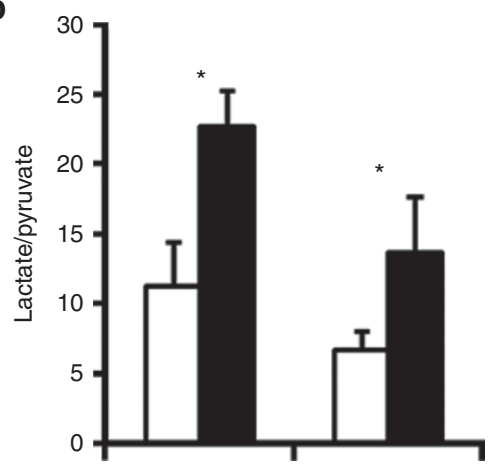

Figure 6. Metabolic switch in retina and choroid in response to hypoxia. (a) Metabolite analysis in hypoxia reveals a shift to glycolysis in choroid (black bars), and a marked mitochondrial impairment in retina (gray bars). Metabolites were measured by mass spectrometry or biochemical assay (see Methods). Values given in hypoxia are expressed as a percentage of values from normoxia group. (b) Lactate-to-pyruvate ratio after hypoxia shows accumulation of lactate in both retina (left) and choroid (right). ${ }^{*} P$ $<0.05,{ }^{* *} P<0.01$. White bars represent normoxia; black bars represent hypoxia.

Despite we cannot exclude that transcriptional regulation of HIF- $1 \alpha$ irrespective of its stabilization may be taking place in the neural tissues that we studied, we suggest that other hypoxia-sensitive transcription factors-like NF- $\mathrm{\kappa B}$ or STAT3 $(25,26)$ might be more relevant in this context.

$\mathrm{NF}-\mathrm{KB}$ is a family of transcription factors that promote crosstalk between hypoxia and inflammation, accomplished by transcriptional regulation of VEGFA and HIF-1A mRNA $(8,18)$. We observed a significant increase in $\mathrm{p} 65$ phosphorylation (subunit of NF- $\mathrm{kB}$ ) in the retina, along with an increase in VEGFA and HIF-1A gene expression. Accordingly, an acute hypoxic episode triggers NF- $\kappa \mathrm{B}$ activation in retinal cells in vitro and in vivo $(18,27)$ and HIF-1A mRNA accumulates in rat brain in response to hypoxia (28). Since NF-kB activation is a common event that links several stress and survival signaling pathways (29), we propose that NF- $\mathrm{KB}$ might be a key regulator of the retinal stress response after hypoxia. Conversely, choroid does not promote p65 phosphorylation or HIF-1A transcription, which is consistent with a blunted or absent stress response in this tissue. A deeper understanding of p65 activation in the hypoxic eye might allow therapeutic intervention in perinatal asphyxia.

Hypoxia, especially if accompanied by ischemia, rapidly exhausts mitochondrial energy substrate availability. Since glycogen stores in the retina are limited, rapid progression to energy collapse will occur during prolonged hypoxia (for review, see ref. (30)). During hypoxia, choroid transitions to a metabolic state characterized by increased anaerobic glycolysis, which leads to lactate accumulation. Metabolite levels in retina suggest a similar metabolic response. Conversely to choroid, a severe reduction of mitochondrial metabolites in retina may rapidly compromise cell survival during hypoxia. Interestingly, the increased lactate production in the retina may arise from an increased metabolic rate from Müller glial cells. Lactate shuttling from Müller glial cells to photoreceptors has been described to fuel mitochondrial oxidative metabolism and glutamate resynthesis in these cells $(31,32)$. Thus, as it occurs from astrocytes to neurons, it is likely that transport of lactate to the neighboring cell is part of a symbiotic mechanism that favors metabolic competence in adverse (or energy demanding) conditions, in this specific case under an hypoxic event.

We found that neuroprotective signals, like p38 activation, $B D N F$ mRNA expression, and HIF- $1 \alpha$ accumulation $(8,22)$ play a minor role in the eye after hypoxia. These mechanisms of cell survival might have a more important role in neuroprotection after long-lasting hypoxia or during reoxygenation.

Tissue adaptation to hypoxia requires changes in blood flow, and eventually compensatory angiogenesis (4). The nature of retinal and choroidal blood flow regulation is probably reflected in the different regulation of gene expression in these tissues. Whereas in the choroid angioneogenesis and vascular permeability are promoted by upregulation of VEGFA, in the retina we found a marked $A D M$ upregulation. ADM likely plays a major role in several ocular conditions, as it is upregulated in glaucoma, diabetic retinopathy, uveitis, and retinitis pigmentosa (33). Despite ADM biology in these pathologies is not fully elucidated, it is known that ADM has pleiotropic activity in the neural tissue (34), which spans from inhibition of macrophage migration and choroidal neovascularization to antioxidant properties in the brain $(35,36)$. Thus, marked increase in $A D M$ in retina might play a key role in retinal physiology, promoting cell survival through diverse mechanisms (37).

Complexity of signaling circuitry and potential role of HIF and NF- $\mathrm{\kappa B}$ transcription factors may help to understand eye (and neural tissue) pathology during hypoxia. Despite our results reflect a preferential activation of NF- $\kappa B$ over HIF immediately after a period of hypoxia, we suggest that a dynamic regulation of both these transcription factors will favor adaptation vs. cell death during prolonged hypoxia or during reoxygenation. Additional studies will be performed to address specifically these questions. 
In summary, our results show that retina engages a very distinct pattern of signaling and transcriptional events compared with the choroid. Major alteration of cellular bioenergetics in the retina may be secondary to a profound $\mathrm{O}_{2}$ deficit. Even under these critical conditions, hypoxia does not trigger an acute activation of pathways of neuroprotection. From a clinical perspective, our findings evidence a specific regional sensitivity to hypoxia, which triggers a different metabolic response in retina and choroid. Preterm brain perfusion, as opposed to term, lacks autoregulation. Hence, in our model choroid could reflect pathophysiologic changes occurring in preterm and retina in term infants respectively and therefore serve as an experimental model for translational purposes (26). On the other hand, the acute pathophysiologic consequences of hypoxia in retina pinpoint a key role for NF- $\kappa B$, which warrants further research. We conclude that retina and choroid can tolerate a period of postnatal hypoxia. Nonetheless, cell survival is transiently achievable due to a metabolic switch, but a more prolonged hypoxic event could be highly detrimental for retinal cell survival.

\section{MATERIALS AND METHODS}

\section{Approval}

The experiments were performed in Norway. The National Animal Research Authority (Norway), approved the experimental protocol. The animals were cared for and handled in accordance with the European Guidelines for Use of Experimental Animals by certified FELASA fellows (Federation of European Laboratory Animals Science Association).

\section{Animal Model and Sample Processing}

A total of 10 newborn Noroc (LyxLD) piglets with the inclusion criteria of age between $12-36 \mathrm{~h}$, hemoglobin $>5 \mathrm{~g} / \mathrm{dl}$ and good general conditions were recruited for the study. The piglets were anesthetized, orally intubated, ventilated, and surgically prepared as previously described by Solberg et al. (38). Complete methods are provided in the Supplementary Methods online.

Five $(n=5)$ piglets were randomly assigned to hypoxia (experimental group $=$ hypoxia $)$ and the remaining five $(n=5)$ to room air (control group $=$ normoxia). At the end of the experiment, when either mean arterial blood pressure had reached $20 \mathrm{mmHg}$ or base excess $-20 \mathrm{mmol} / \mathrm{l}$ and the eyes had been removed, the piglets were given an overdose of $150 \mathrm{mg} / \mathrm{kg}$ of pentobarbital intravenously.

\section{Experimental Protocol}

Hypoxemia, hypotension, and metabolic acidosis were achieved by ventilation with a gas mixture of $8 \% \mathrm{O}_{2}$ in $\mathrm{N}_{2}$ until either mean arterial blood pressure decreased to $20 \mathrm{mmHg}$ or base excess reached $-20 \mathrm{mmol} / \mathrm{l}$. $\mathrm{CO}_{2}$ was added during hypoxemia aiming at a $\mathrm{PaCO}_{2}$ of $8.0-9.5 \mathrm{kPa}$, to imitate perinatal asphyxia and achieving tissue hypoxia-ischemia. The control piglets went through the same procedures, but were not exposed to hypoxia and hypercapnea, They were followed for the same amount of time corresponding to the mean in the hypoxia group. (The duration of the experiment was equal in both groups.) Throughout the whole experiment, there was a continuous surveillance of blood pressure, saturation, pulse, temperature, and blood gas measurements. Hemoglobin was measured on a HemoCue $\mathrm{Hb} 201+($ HemoCue AB, Angelholm, Sweden) at baseline and at the end of the experiment. Temperature-corrected arterial acid/base status, glucose, and lactate were measured regularly throughout the experiment on a Blood Gas Analyzer 860 (Ciba Corning Diagnostics, Midfield, MA). All blood samples obtained from the femoral artery catheter were replaced by normal saline $1.5 \times$ the volume drawn.

\section{Isolation of Retinal and Choroidal Tissue}

The eyeball was proptosed by placing forceps around the optic nerve close to its exit from the eye. From this point on all procedure was performed on ice. A slit was made in the choroid using a small point scissors and the globe was opened. The lens and the corpus vitreum were removed and retina and thereafter choroid were gently separated, transferred to separate vials and snap frozen in liquid nitrogen and stored at $-80^{\circ} \mathrm{C}$ until subsequent analysis.

\section{Metabolites Analysis}

Ultra high performance liquid chromatography tandem mass spectrometry. Tissue samples were homogenized for $1 \mathrm{~min}$ in $\mathrm{N}$ ethyl-maleimide $10 \mathrm{mmol} / \mathrm{l}$ dissolved in phosphate-buffered saline at a $4: 1(\mathrm{v} / \mathrm{w})$, and deproteinized with $4 \%$ acetonitrile $(\mathrm{v} / \mathrm{v})$ followed by centrifugation at $13,000 \mathrm{rpm}$ for $15 \mathrm{~min}, 4^{\circ} \mathrm{C}$. Supernatants were analyzed by a ultra high performance liquid chromatography tandem mass spectrometry system that consisted of an Acquity ultra high performance liquid chromatography coupled to a Xevo TQ-S MS (Waters, Manchester, UK).

Levels of both reduced (GSH) and oxidized (GSSG) glutathione were assessed in retina and choroid matrices from newborn piglets by ultrahigh-performance liquid chromatography tandem mass spectrometry as in Enamoto et al. (39) with slight modifications. In addition, this ultrahigh-performance liquid chromatography method was also set up for simultaneous determination of fructose-1, 6-BP, glucose-6P, (ribose-5P, ribulose-5P), isocitrate, $\alpha$-ketoglutarate, malate, lactate, and pyruvate together GSH and GSSG. MS parameters are provided in the Supplementary Tables S2 and S3 online. Final data for all the metabolites are shown in Supplementary Table S1 online.

Biochemical determination of pyruvate. Pyruvate was determined as conversion of pyruvate to lactate-by-lactate dehydrogenase, by the method of Czok and Lamprecht (40) with minor modifications. Briefly, an aliquot $(50 \mu \mathrm{l})$ of neutralized tricarboxylic acid precipitated sample (or $\mathrm{H}_{2} \mathrm{O}$ for blank determination) was added to the reaction buffer $(10 \mu \mathrm{l}$ of $0.2 \mathrm{mmol} / \mathrm{l} \mathrm{NADH}$ into $200 \mu \mathrm{l}$ of $50 \mathrm{mmol} / \mathrm{l}$ potassium phosphate buffer, $\mathrm{pH}$ 7.4). Spectrophotometric measurement at $340 \mathrm{~nm}$ was performed in a cuvette. After reaching signal stabilization (E1), the reaction was started by addition of $10 \mu \mathrm{l}$ of a lactate dehydrogenase solution ( $1 \mathrm{U})$. Pyruvate concentration was proportional to the difference between absorbance at $30 \mathrm{~min}$ (E2) and E1. Concentration was calculated using a standard curve of sodium pyruvate (range: $0.5-1,000 \mu \mathrm{mol} / \mathrm{l})$.

\section{Real-Time Reverse-Transcriptase PCR}

Total RNA was extracted with TriReagent (Ambion, Austin, TX), and reverse transcribed with RevertAid $\mathrm{H}$ minus First Strand cDNA Synthesis Kit according to the manufacturers' instructions (Fermentas, Glen Burnie, MD). Real-time PCR (iQ5; BioRad, Hercules, CA) was performed with specific primers pairs and SYBR Green as a fluoroflore (Euroclone, Milan, Italy). Gene expression was calculated with $2^{-\triangle \Delta C t}$ method (41), using GAPDH as a reference gene. GAPDH expression level was not different in normoxia vs. hypoxia in choroid (cycle threshold $20.44 \pm 0.54$ vs. $20.68 \pm 1.24, P=0.68$ ) or retina $(17.58 \pm 0.22$ vs. $17.86 \pm 0.28, P=0.11)$. A list of primers used for experiments is presented in Supplementary Table S4 online. Final data for all gene cycle threshold values are shown in Supplementary Table S5 online.

\section{Western Blotting}

Both retina and choroid were homogenized using a mechanical shaker (Precellys; OMNI International, Kennesaw, GA) in radioimmunoprecipitation assay buffer $(50 \mathrm{mmol} / \mathrm{l}$ Tris- $\mathrm{HCl} \mathrm{pH} 7.4,150$ $\mathrm{mmol} / 1 \mathrm{NaCl}, 1 \% \mathrm{NP}-40,0.1 \%$ sodium dodecyl sulfate, $0.5 \%$ sodium deoxycholate, and $1 \mathrm{mmol} / \mathrm{l}$ ethylenediaminetetraacetate) supplemented with phosphatase inhibitors $30 \mathrm{mmol} / \mathrm{l}$ (Na-pyrophosphate, $1 \mathrm{mmol} / \mathrm{l} \mathrm{Na}$-ortovanadate, and $50 \mathrm{mmol} / \mathrm{l} \mathrm{Na}$-fluoride) and protease inhibitors (Sigma, St Louis, MO). Protein samples were quantified by the bicinchoninic acid assay method, following the manufacturer's instructions (Pierce, Rockford, IL).

Samples $(30 \mu \mathrm{g})$ were resolved using a mini-Protean (BioRad) polyacrylamide gel electrophoresis system and electroblotted on nitrocellulose membranes. Blocking was performed in nonfat milk (5\% in Tris-buffered saline-Tween, $1 \mathrm{~h}$,room temperature), primary antibody 
in $5 \%$ bovine serum albumin, and secondary antibody in nonfat milk (5\% in Tris-buffered saline-Tween, $1 \mathrm{~h}$, room temperature). Images were obtained using a CCD camera (BioRad), and densitometric analysis was performed using Image Lab (BioRad). The list of antibodies used in this study is presented in Supplementary Table S6 online.

\section{Statistical Analysis}

Data are presented as mean and SD. Comparison of normoxia to hypoxia was performed by Student's $t$-test. Significant differences were accepted for $P<0.05$.

\section{SUPPLEMENTARY MATERIAL}

Supplementary material is linked to the online version of the paper at http:// www.nature.com/pr

\section{ACKNOWLEDGMENTS}

We would like to express our utmost gratitude to Sylvain Chemtob (Department of Pediatrics and Department of Pharmacology, Research Center-CHU Ste-Justine, Montréal, Quebec, Canada) for his intellectual and conceptual contribution to the hypothesis from which this manuscript originated, and to Frank van Bel (Division of Neonatology, Wilhelmina Children's Hospital/ University Medical Center Utrecht, The Netherlands) for his critical review of the manuscript.

\section{STATEMENT OF FINANCIAL SUPPORT}

This study received financial support in the form of grants Health South-East, Research Foundation, Norway to O.D.S. and Norwegian SIDS and Stillbirth Society (LUB, Landsforeningen Uventet Spedbarnsdød) to R.S., FIS PI1 1/0313 and RD12/0026/0012 (Spanish Ministry of Economy and Competitiveness) to M.V., and CD11/00154 to J.E. (Spanish Ministry of Economy and Competitiveness).

Disclosure: We have nothing to disclose. There were no financial ties to products in this study or potential/perceived conflicts of interest.

\section{REFERENCES}

1. Vento M, Saugstad OD. Resuscitation of the term and preterm infant. Semin Fetal Neonatal Med 2010;15:216-22.

2. Dawson JA, Kamlin CO, Vento M, et al. Defining the reference range for oxygen saturation for infants after birth. Pediatrics 2010;125:e1340-7.

3. Delaey C, Van De Voorde J. Regulatory mechanisms in the retinal and choroidal circulation. Ophthalmic Res 2000;32:249-56.

4. Pournaras CJ, Rungger-Brändle E, Riva CE, Hardarson SH, Stefansson E. Regulation of retinal blood flow in health and disease. Prog Retin Eye Res 2008;27:284-330.

5. Thiersch M, Raffelsberger W, Frigg R, et al. Analysis of the retinal gene expression profile after hypoxic preconditioning identifies candidate genes for neuroprotection. BMC Genomics 2008;9:73.

6. Shahidi M, Wanek J, Blair NP, Mori M. Three-dimensional mapping of chorioretinal vascular oxygen tension in the rat. Invest Ophthalmol Vis Sci 2009;50:820-5.

7. Wanek J, Teng PY, Blair NP, Shahidi M. Inner retinal oxygen delivery and metabolism under normoxia and hypoxia in rat. Invest Ophthalmol Vis Sci 2013;54:5012-9.

8. Fan X, Heijnen CJ, van der Kooij MA, Groenendaal F, van Bel F. The role and regulation of hypoxia-inducible factor-1alpha expression in brain development and neonatal hypoxic-ischemic brain injury. Brain Res Rev 2009;62:99-108.

9. Benita Y, Kikuchi H, Smith AD, Zhang MQ, Chung DC, Xavier RJ. An integrative genomics approach identifies Hypoxia Inducible Factor-1 (HIF1)-target genes that form the core response to hypoxia. Nucleic Acids Res 2009;37:4587-602.

10. Kaelin WG Jr, Ratcliffe PJ. Oxygen sensing by metazoans: the central role of the HIF hydroxylase pathway. Mol Cell 2008;30:393-402.

11. Mahon PC, Hirota K, Semenza GL. FIH-1: a novel protein that interacts with HIF-1alpha and VHL to mediate repression of HIF-1 transcriptional activity. Genes Dev 2001;15:2675-86.
12. Minet E, Arnould T, Michel G, Roland I, Mottet D, Raes M, Remacle J, Michiels C. ERK activation upon hypoxia: involvement in HIF-1 activation. FEBS Lett 2000;468:53-58.

13. Brugarolas J, Kaelin WG Jr. Dysregulation of HIF and VEGF is a unifying feature of the familial hamartoma syndromes. Cancer Cell 2004;6: 7-10.

14. Lendahl U, Lee KL, Yang H, Poellinger L. Generating specificity and diversity in the transcriptional response to hypoxia. Nat Rev Genet 2009;10:82132.

15. Nishijima K, Ng YS, Zhong L, et al. Vascular endothelial growth factorA is a survival factor for retinal neurons and a critical neuroprotectant during the adaptive response to ischemic injury. Am J Pathol 2007;171: 53-67.

16. Kaur C, Sivakumar V, Foulds WS, Luu CD, Ling EA. Cellular and vascular changes in the retina of neonatal rats after an acute exposure to hypoxia. Invest Ophthalmol Vis Sci 2009;50:5364-74.

17. Ergorul C, Ray A, Huang W, Wang DY, Ben Y, Cantuti-Castelvetri I, Grosskreutz CL. Hypoxia inducible factor-1 (HIF-1) and some HIF-1 target genes are elevated in experimental glaucoma. J Mol Neurosci 2010;42:183-191.

18. Lukiw WJ, Ottlecz A, Lambrou G, et al. Coordinate activation of HIF-1 and NF-kappaB DNA binding and COX-2 and VEGF expression in retinal cells by hypoxia. Invest Ophthalmol Vis Sci 2003;44:4163-70.

19. Yokogami K, Yamashita S, Takeshima H. Hypoxia-induced decreases in SOCS3 increase STAT3 activation and upregulate VEGF gene expression. Brain Tumor Pathol 2013;30:135-43.

20. Greisen G. Autoregulation of cerebral blood flow in newborn babies. Early Hum Dev 2005;81:423-8.

21. Moroz E, Carlin S, Dyomina K, et al. Real-time imaging of HIF-1alpha stabilization and degradation. PLoS One 2009;4:e5077.

22. Yang H, Feng GD, Liang Z, et al. In vitro beneficial activation of microglial cells by mechanically-injured astrocytes enhances the synthesis and secretion of BDNF through p38MAPK. Neurochem Int 2012;61: $175-86$.

23. Li L, Qu Y, Li J, Xiong Y, Mao M, Mu D. Relationship between HIF-1alpha expression and neuronal apoptosis in neonatal rats with hypoxia-ischemia brain injury. Brain Res 2007;1180:133-9.

24. Skappak C, Regush S, Cheung PY, Adamko DJ. Identifying hypoxia in a newborn piglet model using urinary NMR metabolomic profiling. PLoS One 2013;8:e65035.

25. Siddiq A, Aminova LR, Ratan RR. Prolyl 4-hydroxylase activity-responsive transcription factors: from hydroxylation to gene expression and neuroprotection. Front Biosci 2008;13:2875-87.

26. Wang H, Byfield G, Jiang Y, Smith GW, McCloskey M, Hartnett ME. VEGF-mediated STAT3 activation inhibits retinal vascularization by down-regulating local erythropoietin expression. Am J Pathol 2012;180: 1243-53.

27. Tirziu D, Jaba IM, Yu P, et al. Endothelial nuclear factor- $\kappa B-$ dependent regulation of arteriogenesis and branching. Circulation 2012;126: 2589-600.

28. Bergeron M, Yu AY, Solway KE, Semenza GL, Sharp FR. Induction of hypoxia-inducible factor-1 (HIF-1) and its target genes following focal ischaemia in rat brain. Eur J Neurosci 1999;11:4159-70.

29. Perkins ND. Integrating cell-signalling pathways with NF-kappaB and IKK function. Nat Rev Mol Cell Biol 2007;8:49-62.

30. Niemeyer G. Retinal research using the perfused mammalian eye. Prog Retin Eye Res 2001;20:289-318.

31. Poitry S, Poitry-Yamate C, Ueberfeld J, MacLeish PR, Tsacopoulos M. Mechanisms of glutamate metabolic signaling in retinal glial (Müller) cells. J Neurosci 2000;20:1809-21.

32. Poitry-Yamate CL, Poitry S, Tsacopoulos M. Lactate released by Müller glial cells is metabolized by photoreceptors from mammalian retina. J Neurosci 1995;15:5179-91.

33. Blom J, Giove TJ, Pong WW, Blute TA, Eldred WD. Evidence for a functional adrenomedullin signaling pathway in the mouse retina. Mol Vis 2012;18:1339-53. 


\section{Articles $\mid$ Arduiniet al.}

34. Kato $\mathrm{H}$, Shichiri M, Marumo F, Hirata Y. Adrenomedullin as an autocrine/ paracrine apoptosis survival factor for rat endothelial cells. Endocrinology 1997;138:2615-20.

35. Yuda $\mathrm{K}$, Takahashi $\mathrm{H}$, Inoue $\mathrm{T}$, et al. Adrenomedullin inhibits choroidal neovascularization via CCL2 in the retinal pigment epithelium. Am J Pathol 2012;181:1464-72.

36. Fujita M, Kuwaki T, Ando K, Fujita T. Sympatho-inhibitory action of endogenous adrenomedullin through inhibition of oxidative stress in the brain. Hypertension 2005;45:1165-72.

37. Chaung WW, Wu R, Ji Y, et al. Peripheral administration of human adrenomedullin and its binding protein attenuates stroke-induced apoptosis and brain injury in rats. Mol Med 2011;17:1075-83.
38. Solberg R, Enot D, Deigner HP, et al. Metabolomic analyses of plasma reveals new insights into asphyxia and resuscitation in pigs. PLoS One 2010;5:e9606

39. Enomoto M, Gosal K, Cubells E, et al. Sex-dependent changes in the pulmonary vasoconstriction potential of newborn rats following short-term oxygen exposure. Pediatr Res 2012;72:468-78.

40. Czok R, Lamprecht W. Pyruvate, Phosphoenolpyruvate and D-glycerate in Methods of Enzymatic Analysis. In Bergmeyer H, Gawehn K, eds. Methods of Enzymatic Analysis. Verlag Chemie: Weinheim Germany, 1974:1446-51.

41. Schmittgen TD, Livak KJ. Analyzing real-time PCR data by the comparative C(T) method. Nat Protoc 2008;3:1101-8. 